\title{
Implementation and Evaluation of a Simulation System Based on Particle Swarm Optimization for Node Placement Problem in Wireless Mesh Networks
}

\author{
Shinji Sakamoto*, Tetsuya Oda \\ Graduate School of Engineering, \\ Fukuoka Institute of Technology, \\ 3-30-1 Wajiro-Higashi, Higashi-Ku, Fukuoka 811-0295, Japan \\ E-mail: shinji.t.sakamoto@gmail.com,oda.tetsuya.fit@gmail.com \\ *Corresponding author
}

\section{Makoto Ikeda, Leonard Barolli}

Department of Information and Communication Engineering

Fukuoka Institute of Technology (FIT)

3-30-1 Wajiro-Higashi, Higashi-Ku, Fukuoka 811-0295, Japan

E-mail: makoto.ikd@acm.org, barolli@fit.ac.jp

\section{Fatos Xhafa}

Technical University of Catalonia

Department of Languages and Informatics Systems

C/Jordi Girona 1-3, 08034 Barcelona, Spain

E-mail: fatos@1si.upc.edu

\begin{abstract}
With the fast development of wireless technologies, Wireless Mesh Networks (WMNs) are becoming an important networking infrastructure due to their low cost and increased high speed wireless Internet connectivity. This paper implements a simulation system based on Particle Swarm Optimization (PSO) in order to solve the problem of mesh router placement in WMNs. 4 replacement methods of mesh routers are considered: Constriction Method (CM), Random Inertia Weight Method (RIWM), Linearly Decreasing Vmax Method (LDVM) and Linearly Decreasing Inertia Weight Method (LDIWM). Simulation results are provided, showing that the CM converges very fast, but has the worst performance among the methods. The considered performance metrics are the Size of Giant Component (SGC) and the Number of Covered Mesh Clients (NCMC). The RIWM converges fast and the performance is good. The LDIWM is a combination of RIWM and LDVM. The LDVM converges after 170 number of phases but has a good performance.
\end{abstract}

Keywords: Wireless Mesh Networks, Particle Swarm Optimization, Node Placement, Giant Component, Client Coverage 


\section{Introduction}

Wireless networks are becoming increasingly popular and they provide users access to information and communication anytime and anywhere Ikeda (2012a), Boyinbode et al. (2011), Kulla et al. (2014), Inaba et al. (2014), Koyama et al. (2011), Hiyama et al. (2012, 2013), Spaho et al. (2011), Ikeda et al. (2015), Goto et al. (2013), Xhafa et al. $(2012 b, a)$, Aikebaier et al. (2011), Ikeda (2012b). Wireless Mesh Networks (WMNs) are have attracted considerable attention due to their low cost nature and their ability to enable wireless Internet connectivity. A WMN is a dynamically self-organized and selfconfigured network, in which the nodes establish and maintain a mesh connectivity among themselves by creating an ad hoc network. This feature brings many advantages to WMNs, including low up-front cost, easy network maintenance, robustness and reliable service coverage Akyildiz et al. (2005). Moreover, thanks to its infrastructure, WMNs have been proven to be very suitable for the deployment of various types of networks and systems in many real-life applications scenarios Vural et al. (2013), the more prominent of which are urban sensing, transportation monitoring, surveillance system deployment, medical system monitoring, to name a few.

Mesh node placement in WMN can be viewed as a set of placement problems which have been addressed using techniques emanated from graph theory or other optimization approaches Muthaiah \& Rosenberg (2008), Franklin \& Murthy (2007). Most of these problem formulations have been found to be computationally hard to solve, calling for the design of heuristics to derive near optimal solutions in some cases.

(a) the locations of mesh router nodes are not pre-determined (any available position in the considered area can be used for deploying the mesh routers) and (b) it is assumed that the routers possess their own radio coverage area. In this paper, the mesh router nodes placement problem is considered, assuming that an area is given where a number of mesh router nodes are deployed, along with a number of mesh client nodes of fixed positions (of an arbitrary distribution) in the area. The objective is to find a location assignment for the mesh routers to the cells of the area that maximizes the network connectivity and client coverage.

Node placement problems are known to be computationally hard to solve Lim et al. (2004), Maolin et al. (2009), Wang et al. (2007). In some previous works, intelligent algorithms have been recently investigated Sakamoto et al. (2015), Barolli et al. (2011), Xhafa et al. (2007), Hoshi et al. (2013), Xhafa et al. (2009), Sakamoto et al. (2014), Girgis et al. (2014), Oda et al. (2012b), Sakamoto et al. (2013), Oda et al. (2012a), Amaldi et al. (2008).

In this paper, a simulation system based on Particle Swarm Optimization (called WMN-PSO) is proposed to solve the mesh router problem in WMNs. We consider 4 replacement methods of mesh routers: CM, RIWM, LDVM and LDIWM. The metrics used for optimization are the Size of Giant Component (SGC) and the Number of Covered Mesh Clients (NCMC).

The rest of the paper is organized as follows. The mesh router nodes placement problem is described in Section 2. In Section 3, our proposed WMN-PSO simulation system is presented. In Section 4, the simulation results are discussed. Finally, Section 5 concludes the paper. 


\section{Node Placement Problem in WMNs}

To tackle the node placement problem in WMN, it is assumed that an area is available and is arranged in cells. The goal is to figure out where to distribute a number of mesh router nodes and a number of mesh client nodes of fixed positions (of an arbitrary distribution) in that area.

Therefore, the objective is to find a location assignment for the mesh routers to the area that maximizes the network connectivity and client coverage. In this problem, the network connectivity is measured by the Size of Giant Component (SGC) of the resulting WMN graph. On the other hand, the user coverage is determined by the number of mesh client nodes that fall within the radio coverage of at least one mesh router node. It is measured by the Number of Covered Mesh Clients (NCMC). The SGC and NCMC will be considered as performance metrics when evaluating the proposed WMN-PSO system using the aforementioned four replacement methods.

An instance of the problem consists of the following.

- $N$ mesh router nodes, each having its own radio coverage. This defines a vector composed of routers

- An area of dimension $W \times H$ where the $N$ routers are to be distributed. It should be noted that the positions of the mesh routers are not predetermined. Rather, these positions are to be computed.

- $M$ client mesh nodes located in arbitrary points of the considered area. This defines a matrix of clients

It should be noted that the network connectivity and user coverage are among the most important metrics in WMNs which directly affect the network performance.

In this work, the node placement problem in WMNs is formulated as a bi-objective optimization problem, where the network connectivity of the WMN is maximized, yielding the optimum SGC, then followed by the maximization of the NCMC.

In fact, one can formalize an instance of the problem by constructing an adjacency matrix associated with the WMN graph, whose nodes are router nodes and client nodes, and whose edges are links between the nodes in the mesh network. Each mesh node in the graph is a triple $v=\langle x, y, r\rangle$ representing the 2D location point $(x, y)$ and radius $r$ is the radius of the transmission range. There is an arc between two nodes $u$ and $v$ if $v$ is within the transmission circular area of $u$.

\section{Proposed WMN-PSO System}

\subsection{PSO}

In PSO Engelbrecht (2005) a number of simple entities (called particles) are placed in the search space of a problem or function, each evaluating the objective function at its current location. The objective function is often minimized and the exploration of the search space is not through evolution Poli et al. (2007). However, following a widespread practice borrowed from the evolutionary computation field, in this work, the bi-objective function and the fitness function are considered interchangeably and each particle determines its movement through the search space by combining some aspects of the history of its own 


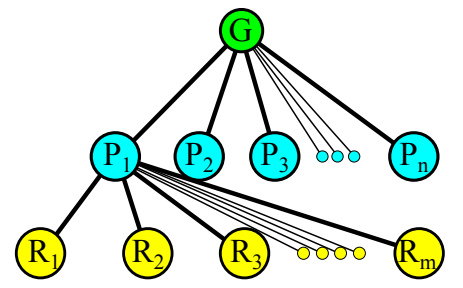

G: Global Solution

P: Particle-pattern

R: Mesh Router

$\mathrm{n}$ : Number of Particle-patterns

m: Number of Mesh Routers

Figure 1 Relationship among global solution, particle-patterns and mesh routers.

current and best fitness locations with those of one or more members of the swarm, with some random perturbations. The next iteration of the algorithm takes place after all the particles have been moved. Eventually, the swarm as a whole will likely move closer to an optimum of the fitness function.

Each individual in the particle swarm is composed of three $\mathcal{D}$-dimensional vectors, where $\mathcal{D}$ is the dimensionality of the search space. These are the current position $\vec{x}_{i}$, the previous best position $\vec{p}_{i}$ and the velocity $\vec{v}_{i}$.

The particle swarm is more than just a collection of particles. A particle by itself has almost no power to solve any problem; progress occurs only when the particles interact. The problem solving is a population-wide phenomenon emerging from the individual behaviors of the particles through their interactions. In any case, populations are organized according to some communication structure or topology, often thought of as a social network. The topology typically consists of bidirectional edges connecting pairs of particles, so that if $j$ is in $i$ 's neighborhood, $i$ is also in $j$ 's neighborhood. Each particle communicates with some other particles and is affected by the best point found by any member of its topological neighborhood. This is just the vector $\vec{p}_{i}$ for that best neighbor denoted as $\vec{p}_{g}$. The potential kinds of population "social networks" are hugely varied, but in practice, certain types of population have been used more frequently.

In the PSO process, the velocity of each particle is iteratively adjusted in such a way that the particle stochastically oscillates around $\vec{p}_{i}$ and $\vec{p}_{g}$ locations.

\subsection{WMN-PSO System for Mesh Router Node Placement}

A new simulator called WMN-PSO is designed and implemented, which uses the PSO algorithm to solve the node placement problem in WMNs. Our system can generate instances of the problem using different iterations of the clients and mesh routers.

The details of the proposed PSO algorithm (referred to as Algorithm 1) for the mesh router placement problem in WMNs are as follows:

\section{Initialization}

Our proposed system starts by generating an initial solution randomly, by using ad hoc methods Xhafa et al. (2009). The velocity of particles is determined a random process considering the area size. For instance, when the area size is $W \times H$, the velocity is decided randomly from $-\sqrt{W^{2}+H^{2}}$ to $\sqrt{W^{2}+H^{2}}$. 


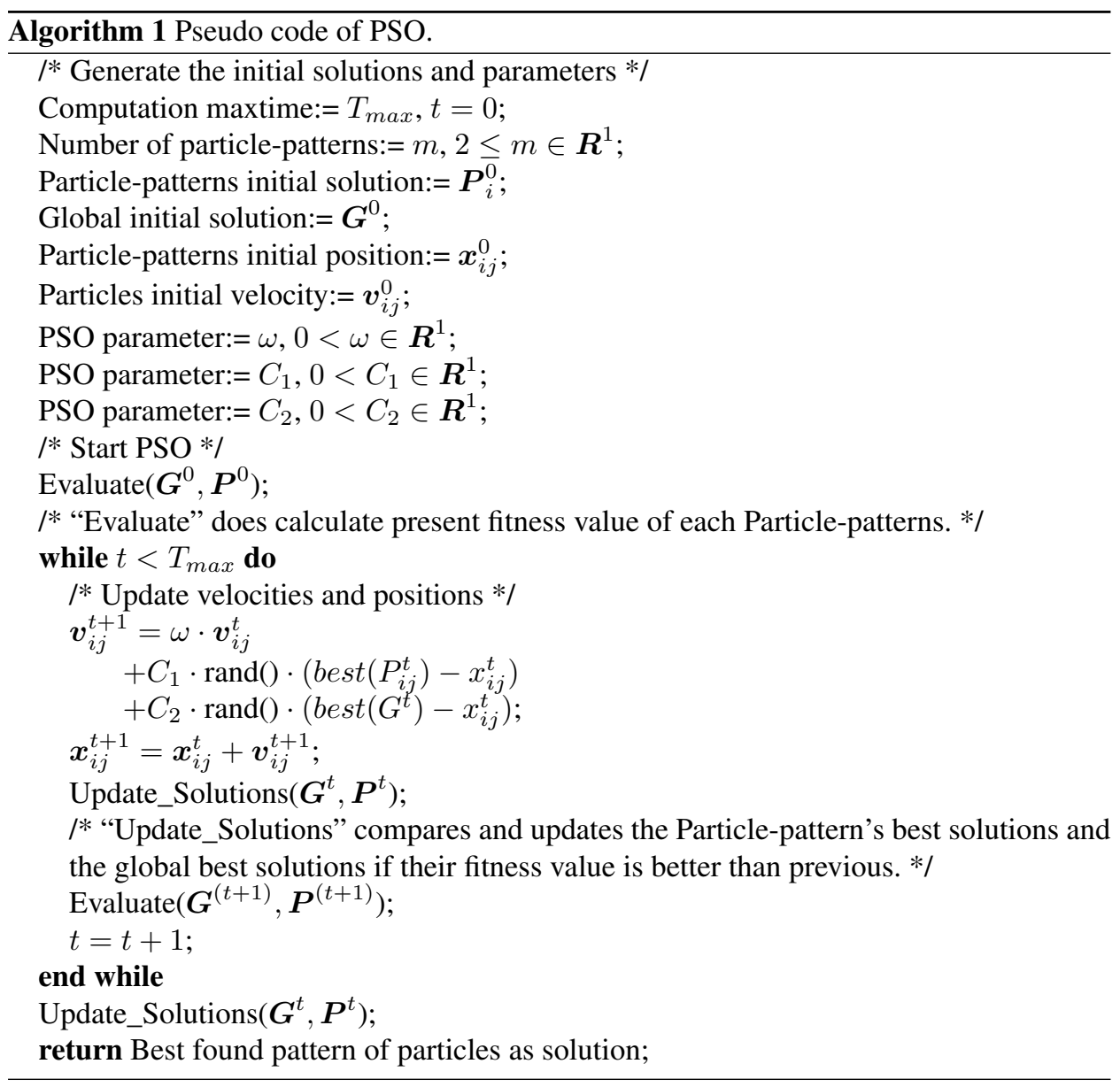

\section{Particle-pattern}

A particle is a mesh router. A fitness value of a particle-pattern is computed by a combination of mesh routers and mesh clients positions, i.e., each particle-pattern is a solution as shown is Fig. 1. Therefore, the number of particle-patterns is a number of solutions.

\section{Fitness function}

One of the main challenges in designing a PSO algorithm is to decide on which appropriate objective function should be considered as well as its encoding. In our case, each particlepattern has its own fitness value which is to be compared against other particle-pattern's fitness value in order to share the information on the global solution. The fitness function follows a hierarchical approach in which the main objective is to maximize the SGC in WMN. Thus, the fitness function of this scenario is considered as

$$
\text { Fitness }=0.7 \times \operatorname{SGC}\left(\boldsymbol{x}_{i j}, \boldsymbol{y}_{i j}\right)+0.3 \times \operatorname{NCMC}\left(\boldsymbol{x}_{i j}, \boldsymbol{y}_{i j}\right) .
$$


Table 1 Simulation parameters.

\begin{tabular}{cc}
\hline Parameters & Values \\
\hline \hline Clients distribution & Normal distribution \\
Area size & $32 \times 32$ \\
Number of mesh routers & 16 \\
Number of mesh clients & 48 \\
Total iterations & 6400 \\
Iteration per phase & 32 \\
Number of particle-patterns & 32 \\
Radius of a mesh router & From $1.5 \times 1.5$ to $4.5 \times 4.5$ \\
Initial router placement & Random \\
Movement methods & CM, RIWM, LDVM, LDIWM \\
\hline
\end{tabular}

\section{Routers replacement method}

A mesh router has $x, y$ positions and velocity. Mesh routers are moved based on velocities. In this paper, the following moving methods in PSO have been considered:

\section{Constriction Method (CM)}

In this method, the PSO parameters are set to a week stable region $\left(\omega=0.729, C_{1}=\right.$ $C 2=1.4955$ ) based on an analysis of the PSO by Clerc \& Kennedy (2002), Shi (2004).

\section{Random Inertia Weight Method (RIWM)}

In this method, the $\omega$ parameter is changing randomly from 0.5 to 1.0 . The $\mathrm{C} 1$ and $\mathrm{C} 2$ are kept 2.0. The $\omega$ can be estimated by the week stable region, yielding an average value of 0.75 Shi (2004).

\section{Linearly Decreasing Vmax Method (LDVM)}

In this method, the PSO parameters are set to an unstable region ( $\omega=0.9, C_{1}=C_{2}=$ 2.0 ). A value of $V_{\max }$ which is the maximum velocity of particles is considered. When the number of iterations (hence computations) increases, $V_{m} a x$ is shown to decrease linearly Schutte \& Groenwold (2005).

\section{Linearly Decreasing Inertia Weight Method (LDIWM)}

In this method, the PSO parameters, $C_{1}$ and $C_{2}$ are set to constant value 2 . On the other hand, the $\omega$ parameter is changed linearly from the unstable region $(\omega=0.9)$ to the stable region $(\omega=0.4)$ when the number of iterations (hence computations) increases Shi (2004), Shi \& Eberhart (1998).

\section{Simulation Results}

This section is devoted to the performance evaluation of our proposed WMN-PSO system. In this work, a $32 \times 32$ area is considered, along with the Normal distribution of mesh clients. The number of mesh routers is set to 16 and the number of mesh clients is set to 48 . We use four different replacement methods of routers: CM, RIWM, LDVM and LDIWM. The total number of iterations is set to 6400 and the iterations per phase is to be 32 . We 

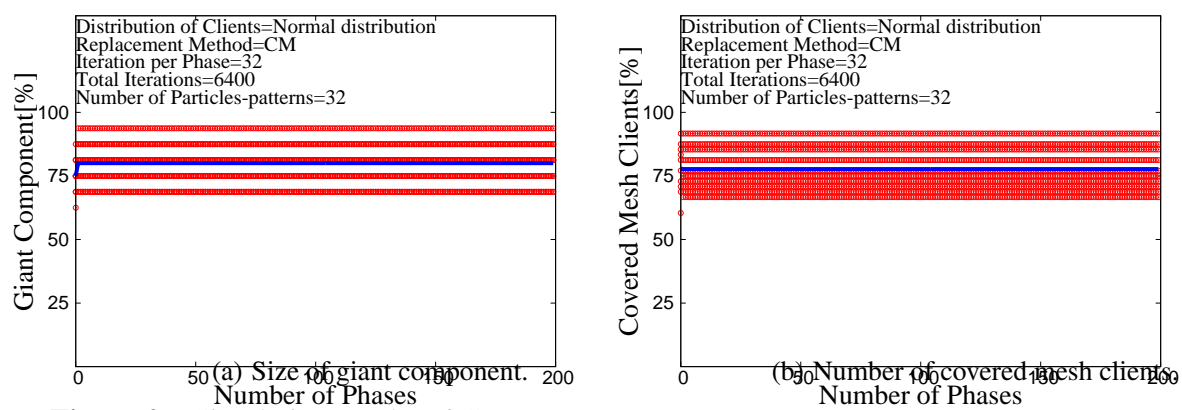

Figure 2 Simulation results of $\mathrm{CM}$.
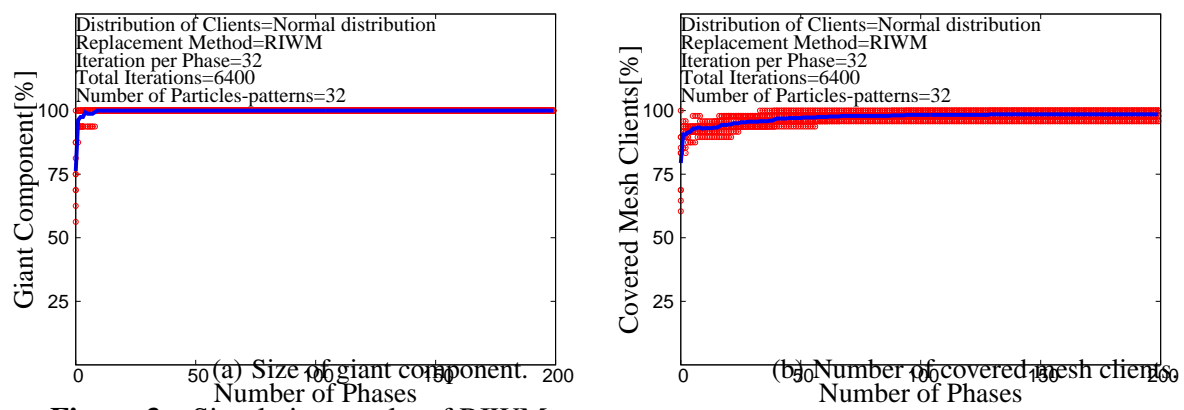

Figure 3 Simulation results of RIWM.
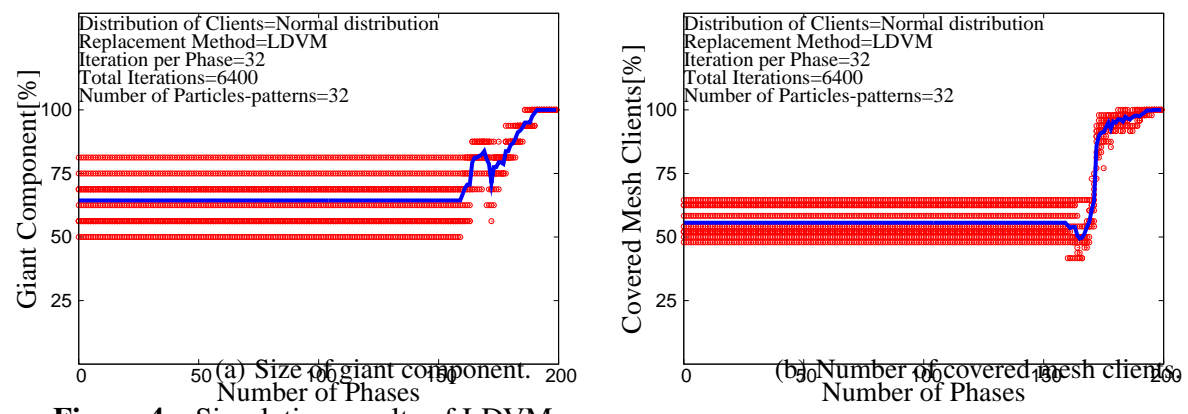

Figure 4 Simulation results of LDVM.

consider that the number of particle-patterns is 32 . The simulation parameters and their values are given in Table 1. The simulations have been conducted 10 times in order to avoid dealing with the effects of randomness and create a general view of the results.

Simulation results are shown in Fig. 2 to Fig. 4. The results of CM are shown in Fig. 2. For CM, the performance is almost constant. Its convergence is very fast, but the performance is not good. The results of RIWM are shown in Fig. 3. In RIWM, the particle- 

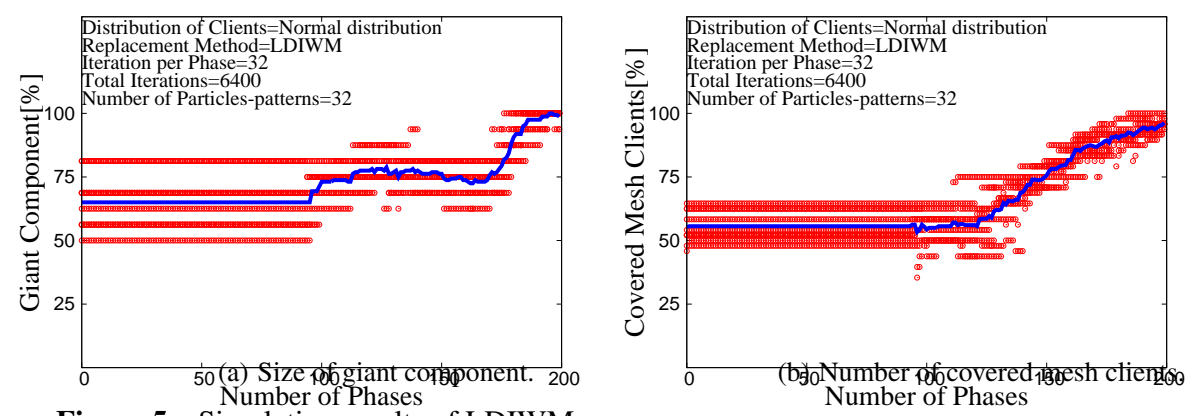

Figure 5 Simulation results of LDIWM.

patterns converge to a good solution fast and the performance is very good. The simulation results of LDVM are shown in Fig. 4. In LDVM, the solutions converge after 170 phases. In Fig. 5, we show the simulation results when the replacement method is LDIWM. The particle-patterns converge after 200 phases.

In Fig. 6, the visualization results for the four replacement methods for mesh routers are shown. In Fig. 6(a), for CM, it can be observed that some of mesh routers create an island and do not cover any mesh client. Other 3 replacement methods except the CM can find good solutions because all mesh routers connect to all mesh clients by using multi hops.

Comparing the four different replacement methods, it can be concluded that:

- The CM converges very fast but it has the worst performance among 4 replacement methods.

- The RIWM converges fast and has a good performance.

- The LDVM converges after 170 phases and it has a good performance.

- The LDIWM converges after 200 phases.

\section{Conclusions}

In this work, we have implemented a simulation system using PSO in order to solve the mesh router placement problem in WMNs. We considered four replacement methods for mesh routers. Using the Size of Giant Component (SGC) and the Number of Covered Mesh Clients (NCMC) as performance metrics, our simulation have revealed that:

- The CM converges very fast but it has the worst performance among 4 replacement methods.

- The RIWM converges fast and has a good performance.

- The LDVM converges after 170 phases and it has a good performance.

- The LDIWM converges after 200 phases. 


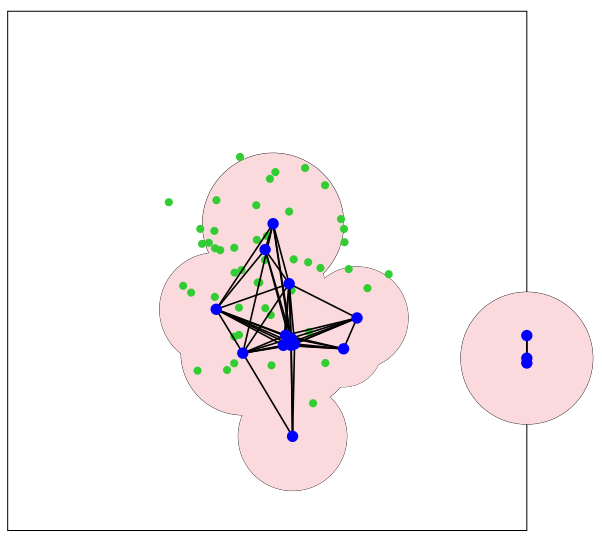

(a) CM.

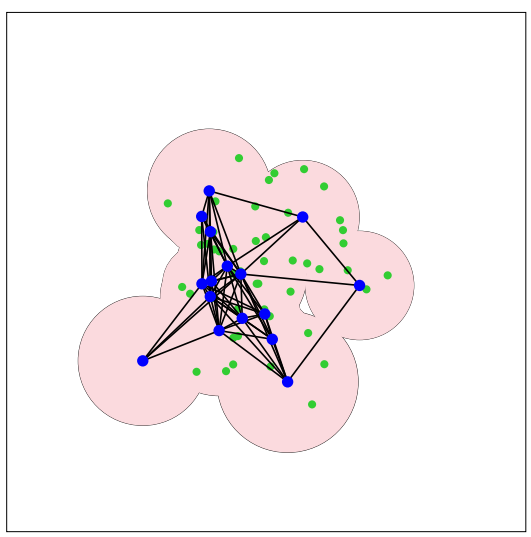

(c) LDVM

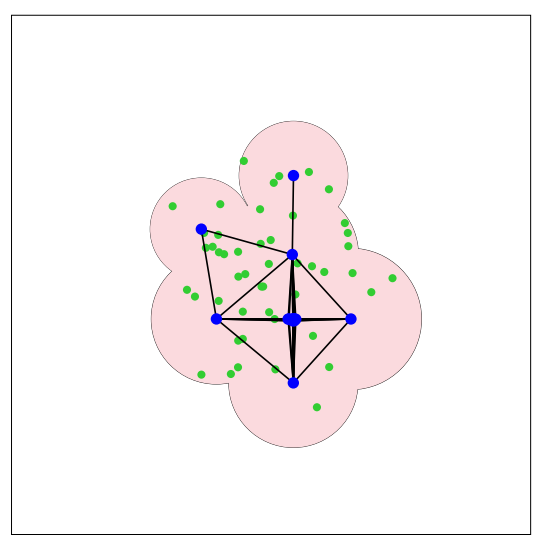

(b) RIWM.

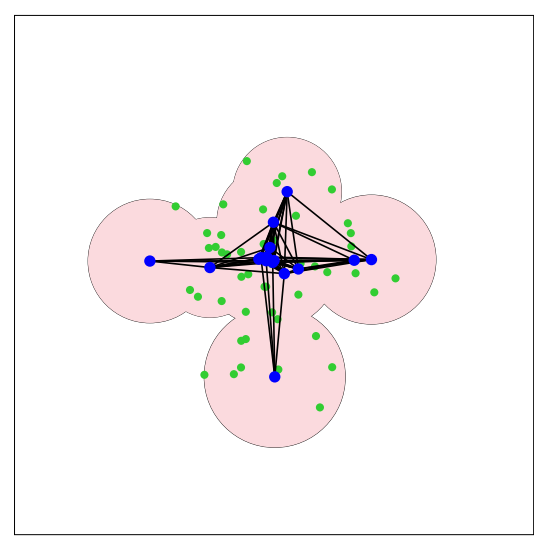

(d) LDIWM.

Figure 6 Visualization of results.

In our future work, we would like to evaluate the performance of the proposed system for different other parameters and patterns. Moreover, it would also be desirable to compare its performance with other learning algorithms such as stochastic learning automatic algorithms, ant colony optimization algorithms, to name a few.

\section{References}

Aikebaier, A., Enokido, T. \& Takizawa, M. (2011), 'TMPR-scheme for Reliably Broadcast Messages Among Peer Processes', International Journal of Grid and Utility Computing 2(3), 175-182.

Akyildiz, I. F., Wang, X. \& Wang, W. (2005), 'Wireless Mesh Networks: A Survey', Computer Networks 47(4), 445-487.

Amaldi, E., Capone, A., Cesana, M., Filippini, I. \& Malucelli, F. (2008), 'Optimization Models and Methods for Planning Wireless Mesh Networks', Computer Networks 52(11), 2159-2171. 
Barolli, A., Spaho, E., Barolli, L., Xhafa, F. \& Takizawa, M. (2011), 'QoS Routing in Ad-hoc Networks using GA and Multi-objective Optimization', Mobile Information Systems 7(3), 169188.

Boyinbode, O., Le, H. \& Takizawa, M. (2011), 'A Survey on Clustering Algorithms for Wireless Sensor Networks', International Journal of Space-Based and Situated Computing 1(2), 130 136.

Clerc, M. \& Kennedy, J. (2002), 'The Particle Swarm-Explosion, Stability, and Convergence in a Multidimensional Complex Space', IEEE Transactions on Evolutionary Computation 6(1), 58 73.

Engelbrecht, A. P. (2005), Fundamentals of Computational Swarm Intelligence, Wiley \& Sons.

Franklin, A. A. \& Murthy, C. S. R. (2007), 'Node Placement Algorithm for Deployment of Two-tier Wireless Mesh Networks', Proc. of Global Telecommunications Conference pp. 4823-4827.

Girgis, M. R., Mahmoud, T. M., Abdullatif, B. A. \& Rabie, A. M. (2014), 'Solving the Wireless Mesh Network Design Problem using Genetic Algorithm and Simulated Annealing Optimization Methods', International Journal of Computer Applications 96(11), 1-10.

Goto, K., Sasaki, Y., Hara, T. \& Nishio, S. (2013), 'Data Gathering using Mobile Agents for Reducing Traffic in Dense Mobile Wireless Sensor Networks', Mobile Information Systems 9(4), 295314.

Hiyama, M., Kulla, E., Ikeda, M. \& Barolli, L. (2012), 'Evaluation of MANET Protocols for Different Indoor Environments: Results from a Real MANET Testbed', International Journal of Space-Based and Situated Computing 2(2), 71-82.

Hiyama, M., Sakamoto, S., Kulla, E., Ikeda, M. \& Barolli, L. (2013), 'Experimental Results of a MANET Testbed for Different Settings of HELLO Packets of OLSR Protocol', Journal of Mobile Multimedia 9(1-2), 27-38.

Hoshi, T., Kumata, Y. \& Koyama, A. (2013), 'A Proposal and Evaluation of Access Point Allocation Algorithm for Wireless Mesh Networks', International Conference on NetworkBased Information Systems (NBiS-2013) pp. 389-394.

Ikeda, M. (2012a), 'Analysis of Mobile Ad-hoc Network Routing Protocols using Shadowing Propagation Model', International Journal of Space-Based and Situated Computing 2(3), 139148.

Ikeda, M. (2012b), 'End-to-End Single and Multiple Flows Fairness in Mobile Ad-hoc Networks', Journal of Mobile Multimedia 8(3), 204-224.

Ikeda, M., Honda, T. \& Barolli, L. (2015), 'Performance of Optimized Link State Routing Protocol for Video Streaming Application in Vehicular Ad-hoc networks Cloud Computing', Concurrency and Computation: Practice and Experience 27(8), 2054-2063.

Inaba, T., Sakamoto, S., Kulla, E., Caballe, S., Ikeda, M. \& Barolli, L. (2014), 'An Integrated System for Wireless Cellular and Ad-Hoc Networks Using Fuzzy Logic', International Conference on Intelligent Networking and Collaborative Systems (INCoS-2014) pp. 157-162.

Koyama, A., Sato, Y. \& Barolli, L. (2011), 'IEZRP: An Improved Enhanced Zone-based Routing Protocol for MANETs and its Performance Evaluation', International Journal of Space-Based and Situated Computing 1(4), 213-221.

Kulla, E., Mino, G., Sakamoto, S., Ikeda, M., Caballé, S. \& Barolli, L. (2014), 'FBMIS: A Fuzzy-Based Multi-interface System for Cellular and Ad Hoc Networks', IEEE International Conference on Advanced Information Networking and Applications (AINA-2014) pp. 180-185.

Lim, A., Rodrigues, B., Wang, F. \& Xu, Z. (2004), 'k-Center Problems with Minimum Coverage', Computing and Combinatorics pp. 349-359.

Maolin, T. et al. (2009), 'Gateways Placement in Backbone Wireless Mesh Networks', International Journal of Communications, Network and System Sciences 2(1), 44. 
Muthaiah, S. N. \& Rosenberg, C. P. (2008), 'Single Gateway Placement in Wireless Mesh Networks', Proc. of 8th International IEEE Symposium on Computer Networks pp. 4754-4759.

Oda, T., Barolli, A., Spaho, E., Xhafa, F., Barolli, L. \& Takizawa, M. (2012a), 'Evaluation of WMNGA for Different Mutation Operators', International Journal of Space-Based and Situated Computing 2(3), 149-157.

Oda, T., Barolli, A., Xhafa, F., Barolli, L., Ikeda, M. \& Takizawa, M. (2012b), 'Performance Evaluation of WMN-GA for Different Mutation and Crossover Rates Considering Number of Covered Users Parameter', Mobile Information Systems 8(1), 1-16.

Poli, R., Kennedy, J. \& Blackwell, T. (2007), 'Particle Swarm Optimization', Swarm intelligence 1(1), 33-57.

Sakamoto, S., Kulla, E., Oda, T., Ikeda, M., Barolli, L. \& Xhafa, F. (2013), 'A Comparison Study of Simulated Annealing and Genetic Algorithm for Node Placement Problem in Wireless Mesh Networks', Journal of Mobile Multimedia 9(1-2), 101-110.

Sakamoto, S., Kulla, E., Oda, T., Ikeda, M., Barolli, L. \& Xhafa, F. (2014), 'Performance Evaluation Considering Iterations per Phase and SA Temperature in WMN-SA System', Mobile Information Systems 10(3), 321-330.

Sakamoto, S., Oda, T., Ikeda, M., Barolli, L. \& Xhafa, F. (2015), ‘A PSO-based Simulation System for Node Placement in Wireless Mesh Networks: Evaluation Results for Different Replacement Methods', Proc. of The 10th International Conference on Broadband and Wireless Computing, Communication and Applications (BWCCA-2015) pp. 213-219.

Schutte, J. F. \& Groenwold, A. A. (2005), ‘A Study of Global Optimization using Particle Swarms', Journal of Global Optimization 31(1), 93-108.

Shi, Y. (2004), 'Particle Swarm Optimization', IEEE Connections 2(1), 8-13.

Shi, Y. \& Eberhart, R. C. (1998), 'Parameter Selection in Particle Swarm Optimization', Evolutionary programming VII pp. 591-600.

Spaho, E., Mino, G., Barolli, L. \& Xhafa, F. (2011), 'Goodput and PDR Analysis of AODV, OLSR and DYMO Protocols for Vehicular Networks using CAVENET', International Journal of Grid and Utility Computing 2(2), 130-138.

Vural, S., Wei, D. \& Moessner, K. (2013), 'Survey of Experimental Evaluation Studies for Wireless Mesh Network Deployments in Urban Areas Towards Ubiquitous Internet', Communications Surveys \& Tutorials, IEEE 15(1), 223-239.

Wang, J., Xie, B., Cai, K. \& Agrawal, D. P. (2007), 'Efficient Mesh Router Placement in Wireless Mesh Networks', Proc. of IEEE Internatonal Conference on Mobile Adhoc and Sensor Systems (MASS-2007) pp. 1-9.

Xhafa, F., Barolli, L. \& Durresi, A. (2007), 'An Experimental Study on Genetic Algorithms for Resource Allocation on Grid Systems', Journal of Interconnection Networks 8(4), 427-443.

Xhafa, F., Kolodziej, J., Barolli, L., Kolici, V., Miho, R. \& Takizawa, M. (2012a), 'Hybrid Algorithms for Independent Batch Scheduling in Grids', International Journal of Web and Grid Services 8(2), 134-152.

Xhafa, F., Sanchez, C. \& Barolli, L. (2009), 'Ad hoc and Neighborhood Search Methods for Placement of Mesh Routers in Wireless Mesh Networks', Proc. of 29th IEEE International Conference on Distributed Computing Systems Workshops (ICDCS-2009) pp. 400-405.

Xhafa, F., Sun, J., Barolli, A., Biberaj, A. \& Barolli, L. (2012b), 'Genetic Algorithms for Satellite Scheduling Problems', Mobile Information Systems 8(4), 351-377. 